\title{
Power of Plankton: Effects of Algal Biodiversity on Biocrude Production and Stability
}

\author{
Anita Narwani, ${ }^{* \dagger}$ Aubrey R. Lashaway, ${ }^{\ddagger}$ David C. Hietala, ${ }^{\S}$ Phillip E. Savage, ${ }^{\S},{ }_{\odot}$ and Bradley J. Cardinale ${ }^{\ddagger}$ \\ ${ }^{\dagger}$ Department of Aquatic Ecology, Eawag (Swiss Federal Institute of Aquatic Science and Technology), BU-G11 Überlandstrasse 133, \\ 8600 Dübendorf, Switzerland \\ ${ }^{\ddagger}$ School of Natural Resources and Environment, University of Michigan, 1556 Dana Building, 440 Church Street, Ann Arbor, \\ Michigan 48109-1041, United States \\ ${ }^{\S}$ Department of Chemical Engineering, University of Michigan, 3074 H.H. Dow Building, 2300 Hayward Street, Ann Arbor, Michigan \\ 48109, United States \\ "Department of Chemical Engineering, The Pennsylvania State University, 160 Fenske Lab, University Park, Pennsylvania 16802, \\ United States
}

Supporting Information

ABSTRACT: Algae-derived biocrude oil is a possible renewable energy alternative to fossil fuel based crude oil. Outdoor cultivation in raceway ponds is estimated to provide a better return on energy invested than closed photobioreactor systems. However, in these open systems, algal crops are subjected to environmental variation in temperature and irradiance, as well as biotic invasions which can cause costly crop instabilities. In this paper, we used an experimental approach to investigate the ability of species richness to maximize and stabilize biocrude production in the face of weekly temperature fluctuations between 17 and $27{ }^{\circ} \mathrm{C}$, relative to a constant-temperature control. We hypothesized that species richness would lead to higher mean biocrude production and greater stability of biocrude production over time in the variable temperature environment. Counter to our hypothesis, species richness tended to cause a decline in mean biocrude production, regardless of environmental temperature variation. However, biodiversity did have stabilizing effects on biocrude production over time in the variable temperature environment and not in the constant temperature environment. Altogether, our results suggest that when the most productive and stable monoculture is unknown, inoculating raceway ponds with a diverse mixture of algae will tend to ensure stable harvests over time.

\section{INTRODUCTION}

Limited fossil fuel supplies, increasing demand for energy, and increasing atmospheric $\mathrm{CO}_{2}$, have recently brought algaederived biofuels to the forefront of renewable energy research programs. ${ }^{1-5}$ Algae may be a promising potential future source of renewable energy for many reasons. They require relatively little land when compared to other biofuel crops such as soybean, canola, and oil palm. ${ }^{2,3}$ They have short generation times and can be harvested rapidly. They are diverse, so species can be found that tolerate a large range of environmental conditions, and they can be grown on waste streams from industrial and agricultural processes. $^{6-8}$ Only recently have production facilities grown in scale, and start-up companies begun to try to commercialize algal biofuels. ${ }^{1,4,9}$ This is partly because there are still a number of hurdles to be overcome for industrial-scale production to become a reality, and for algae-based fuels to become economically competitive with fossil fuels. We focus here on those hurdles related to the algal biofuel production stream, rather than those related to biomass harvesting or processing. There are currently two main modes of production: closed photobioreactors and open raceway ponds. It is thought that the energy return on investment is greater in raceway ponds due to their lower material and energy costs. ${ }^{4}$ However, because raceway ponds are open, they also suffer from crop instabilities due to environmental variation in light and temperature, as well as due to disturbances from pests, diseases, and unwanted invaders. ${ }^{1,4,6}$ As a result, maximizing and stabilizing raceway pond productivity is a growing focus of algal biofuels research.

To date, most algal biofuels operations are focused on growing monocultures of highly productive species. Synthetic

Revised: October 3, 2016

Accepted: November 9, 2016

Published: November 9, 2016
Received: July 5, 2016 
biology approaches are used to create superspecies or strains that are capable of resisting diseases and consumption, of being more competitive against invaders, of producing more lipid per cell, of photosynthesizing more efficiently, and of tolerating a broader range of environmental conditions. ${ }^{1,4,9}$ However, ecologists, evolutionary biologists, and systems biologists criticize such approaches because there are physical and physiological trade-offs among particular functions that prevent any single organism from performing them all well. ${ }^{10-15}$ Rather than aiming to create a single strain or species that can perform all functions simultaneously, an ecological approach to overcoming these problems would be to design synthetic communities containing multiple species that each performs a separate set of functions. ${ }^{10,15}$ Two decades of research in community ecology has shown that communities containing many species are on average more productive, more efficient at using resources, better at resisting damage from consumers, resisting invasions, and maintaining stable ecosystem functioning over time. ${ }^{16-21}$

Diversity may help to maximize and stabilize biomass or biofuel production in a number of ways. The presence of many species in a community increases the chances that some species in the community will be highly productive (sampling effect sensu Huston ${ }^{22}$ ). Diversity may also enhance biomass production and resource-use efficiency when species coexist via niche partitioning or positive species interactions. ${ }^{23}$ Aggregate community properties such as biomass production and resource-use efficiency may also be stabilized over time in numerous ways. ${ }^{24-32}$ First, when species in high diversity communities coexist via niche partitioning or positive interactions, diversity will increase average levels of community biomass and, in turn, will reduce the influence of stochasticity and likelihood of crashes to low levels. ${ }^{29,33}$ Second, such coexistence will also stabilize individual population-level biomass, which, in turn reduces the summed variance of all species' biomasses. ${ }^{29}$ Last, when species display asynchronous dynamics, either because they respond uniquely to environmental variation, ${ }^{24}$ or because of competitive species interactions, ${ }^{30}$ the total community biomass is stabilized by the presence of a greater number of species; ${ }^{23,24,26,27,30}$ when one species is doing poorly, another is doing well and aggregate properties are less likely to drop below critical levels.

In this study we tested whether algal biodiversity increases the production and stability of algal biocrude oil over time. It has previously been shown that algal diversity may help to stabilize algal biomass in freshwater ecosystems (but see refs 30 and 34). Two early tests showed positive effects of species and functional group diversity on lipid production in freshwater algae. ${ }^{35,36}$ Similarly, Shurin et al. ${ }^{11}$ found that biodiversity had positive effects on the total biovolume of algal communities. Contrary to these examples, however, Bhattacharjee and Siemann ${ }^{37}$ found that monocultures of algae were more productive, stable, and resistant to invasions by other algae than polycultures when growing in wastewater. Key advances of our work over these previous studies are (1) to our knowledge, we provide the first demonstration of the impact of biodiversity on the stability of biocrude production in a fluctuating environment where environmental variability was directly manipulated and (2) we made direct measurements of algal biocrude production using a process known as hydrothermal liquefaction ("HTL", see below), rather than measuring proxies such as lipid production, biomass, or biovolume.

We performed a set of indoor mesocosm experiments designed to test three hypotheses and their consequent predictions regarding the role of diversity in maximizing and stabilizing biocrude production over time in the presence and absence of environmental temperature variation (the predictions further elaborated in the Materials and Methods section). We aimed to test hypotheses that are relevant for outdoor cultivation, but, in order to investigate the role of species diversity per se in response to a particular type of environmental variation (temperature), we set up the experiments in an indoor facility where environmental variation could be well-replicated and controlled. Our first hypothesis was that polycultures would produce greater amounts of biocrude over time on average than monocultures. Our second hypothesis was that polycultures would also display more stable biocrude production over time on than monocultures, particularly in fluctuating environments. Our third hypothesis was that polycultures would better ensure a minimum required biomass threshold for continued pond operations. It is known that monocultures are susceptible to crashes of biomass in outdoor cultivation and that such crashes require pond cleaning, sterilization, and culture restart, ${ }^{4,5,8,38}$ all of which are costly. We assumed that, below this threshold, the algal culture would be considered to have failed, and the pond would be emptied, cleaned, and restarted with a high cost of lost time, material, and energy. Lastly, we used species-specific, population-level biovolume data estimated in monoculture and mixture in order to determine how the mean, variance, and synchrony of individual species contributed to total community biovolume and resultant biocrude production over time.

\section{MATERIALS AND METHODS}

Focal Species Pool. We selected six species for the experiment: Ankistrodesmus falcatus (denoted as " $\mathrm{A}$ " in figures), Chorella sorokiniana ("B"), Pediastrum duplex ("C"), Scenedesmus acuminatus ("D"), Scenedesmus ecornis ("E"), and Selenastrum capricornutum ("F"). We selected these species based on a number of criteria: (1) they were part of the U.S. Department of Energy's Aquatic species program and were included in the Solar Energy Research Program's collection, ${ }^{7}$ (2) they were identified as species of interest for biofuel production in the peer-reviewed literature, ${ }^{39-41}$ (3) they grow on standard culture media (Bold's $3 \mathrm{~N}$ ), and (4) they are morphologically distinguishable under a compound light microscope at $40 \times$ magnification. All six species are also common in lakes across North America based on the EPA's 2007 Lakes Assessment survey (i.e., occurred in at least $15 \%$ of all lakes and were ranked in the top 50 out of 262 genera in occurrence frequency; http://water.epa.gov/type/lakes/NLA data.cfm). Natural occurrence is important because algal biofuels farms that use ecologically based community engineering should not pose a risk of the release, and potential subsequent invasion, of non-native taxa into natural ecosystems.

Predictions and Experimental Design. Based on our first hypothesis, we predicted that the average mixed-species polyculture would have higher biocrude production (averaged over time) than the average monoculture $\left(\overline{\bar{Y}}_{\mathrm{P}}>\overline{\bar{Y}}_{\mathrm{M}}\right)$. Second, we predicted that the best polyculture would provide greater average biocrude production than the best monoculture $\left(\bar{Y}_{\text {best_P }}>\bar{Y}_{\text {best_M }}\right)$. Our focus in this prediction shifts from the average monoculture, to the best monoculture, because an algae farmer is likely to aim to exceed the highest level of production that she/he could obtain from their most productive monoculture, assuming that a single, most productive, species has been identified. Regarding our second hypothesis, we predicted that on average polycultures would show lower variation in biocrude production over time than the average 
monoculture $\left(\overline{\mathrm{CV}}_{\mathrm{P}}<\overline{\mathrm{CV}}_{\mathrm{M}}\right)$. We also predicted that the most stable polyculture would show less variation in biocrude production over time than the most stable monoculture $\left(\mathrm{CV}_{\text {best_P }}<\mathrm{CV}_{\text {best } M}\right)$. Again, this prediction assumes that an algae farmer is able to identify the most stable monoculture for a particular set of environmental conditions, and aims to exceed its stability using mixed culture. We also tested the prediction that polycultures would provide greater stabilizing benefits in a variable environment than in a constant environment due to the chance that diverse communities would contain species that respond uniquely to environmental variation $\left(\left[\overline{\mathrm{CV}}_{\mathrm{M}}-\overline{\mathrm{CV}}_{\mathrm{P}}\right]_{\text {variable }}>\left[\overline{\mathrm{CV}}_{\mathrm{M}}-\overline{\mathrm{CV}}_{\mathrm{P}}\right]_{\text {constant }}\right)$. Lastly, according to our third hypothesis, we tested the prediction that polycultures have a higher probability of maintaining biomasses above the threshold of $30 \mathrm{mg} \cdot \mathrm{L}^{-1}$ than monocultures $\operatorname{Pr}\left[Y_{\mathrm{P}}>T^{*}\right]>\operatorname{Pr}\left[Y_{\mathrm{M}}>T^{*}\right]$. Operational procedures for such cultivation restarts are likely to depend on particular species, local environmental conditions, and pond size and structure. ${ }^{8}$ However, a published estimate indicates that, upon inoculation, the raceway biomass concentration may be as low as $50 \mathrm{mg} \cdot \mathrm{L}^{-1}$. Furthermore, at maximum production, pond productivity should attain an average of $25 \mathrm{mg} \cdot \mathrm{L}^{-1} \cdot \mathrm{d}^{-1}$, with a pond dilution rate of $0.167 \cdot \mathrm{d}^{-1}$, , which means the pond must achieve a standing biomass of at least $150 \mathrm{mg} \cdot \mathrm{L}^{-1}$. Here, we tested our hypothesis under the assumption that ponds must not fall below a biomass concentration of $30 \mathrm{mg} \cdot \mathrm{L}^{-1}$ in order to continue operations. This conservative estimate is $40 \%$ lower than the inoculation concentration of $50 \mathrm{mg} \cdot \mathrm{L}^{-1}$, and it is $80 \%$ lower than the baseline operating biomass concentration of $150 \mathrm{mg} \cdot \mathrm{L}^{-1}$.

The experimental design was a randomized complete block where each combination of two treatments (algal species richness $\times$ temperature variability) were replicated in each of two experimental blocks that were run at different times. The species richness treatment consisted of all possible monocultures of each of the six focal species (replicated 12 times each, six per block), all 15 possible bicultures (replicated eight times each, four per block), 15 randomly chosen four-species mixtures (replicated eight times each, 4 per block), and the full six-species mixture (replicated 18 times, nine per block). Within each block, we used $18010 \mathrm{~L}$ aquaculture tanks that were filled with Bold's $3 \mathrm{~N}$ medium (University of Texas Culture Collection: http://www.utex.org/mediaDetail. aspx?mediaID $=55)$. Bold $3 \mathrm{~N}$ is a nutrient-rich algal growth medium ( $8.82 \mathrm{mM}$ of nitrate and $0.43 \mathrm{mM}$ of phosphate). ${ }^{43}$ Tanks received $30 \%$ media exchanges once a week via an automated pump delivering medium from a central sump (one for each rack) via a series of manifolds and through a backflushing valve and medical drip system attached to each tank. The valve and drip systems prevented back-flow and crosscontamination of liquid between tanks (SI Figure 1). Tanks were each lit from below by four AgroBrite T5 Bulbs (high output, $24 \mathrm{~W}$, full daylight $6400 \mathrm{~K}$ spectrum) set to a light: dark cycle of 18:6. They were continuously bubbled with air through an airstone fed via a manifold attached to an air pump.

The experimental units used in the study were 10-L tanks. There were 180 total tanks that were arranged in six racks containing 30 tanks each (SI Figure 2). Three racks per block were randomly assigned to a constant temperature treatment of $22{ }^{\circ} \mathrm{C}$ (a total of 90 tanks), and three racks per block were assigned to a variable temperature treatment, which fluctuated between 17 and $27{ }^{\circ} \mathrm{C}$ at weekly intervals (SI Table 1). Rack temperature assignments were randomized for each block, and placements of species diversity treatments and compositions were randomized within block and temperature treatments. To maintain temperature treatments, each tank contained a silicone cooling coil, through which temperature-controlled, sterile medium was continuously pumped. The medium, which was continuously UV-sterilized in each rack's central sump, was temperature-controlled via a series of heaters and chillers which were attached to a temperature sensor (SI Figure 2). Media flowed through the silicone coils in each tank and was returned to the sumps for further continuous sterilization and temperature control.

Algal Inoculations. Prior to the experiment, we maintained all species in the lab in batch culture on sterile Bold's $3 \mathrm{~N}$. Prior to inoculation of the experimental units, all tanks and component parts were autoclaved, all plumbing and sumps were bleached, and all media was UV-sterilized. Tanks were filled with $10 \mathrm{~L}$ of Bold's $3 \mathrm{~N}$ media via media-delivery manifolds fed by a pump in each sump on the day of inoculation. Once the tanks were filled, algae were inoculated into their predetermined tank locations at a total density of 12000 cells per milliliter (divided equally among species assigned to polycultures). For colonial species (e.g., Pediastrum duplex), cell density was calculated by estimating colony density and multiplying this by the average number of cells per colony, which we measured for a total of 25 colonies.

Monitoring and Weekly Sampling. After inoculation but before the start of the temperature fluctuations, we monitored the raw chlorophyll-a fluorescence of the four replicate monocultures for all six species every 2-3 days in order to ensure that they had reached a steady-state. Steady-state was achieved when the mean fluorescence of subsequent time points in the time-series of a monoculture were not significantly different from each other (SI Figure 3). After three weeks, we sampled all 180 tanks and began the variable temperature treatments by alternating water temperature from 17 to $27{ }^{\circ} \mathrm{C}$ at 7 -day intervals. We continued sampling weekly for six more weeks (7 weeks in total).

Weekly sampling of the tanks consisted of removing $3 \mathrm{~L}$ of culture media from every tank via sterile silicone tubing attached to ports on the front of the tanks. A $2.5 \mathrm{~L}$ subsample was allowed to settle for 1 week at room temperature. After settling, $\sim 90 \%$ of the liquid was removed from the tops of the bottles using peristaltic pumps (Cole-Parmer Masterflex L/S Model no. 7523-80). The algal slurry remaining was then centrifuged, decanted and dried for 1 week at $60^{\circ} \mathrm{C}$, to achieve a dry algal biomass pellet. This pellet was used for the HTL reactions (see HTL Measurement of Biocrude below).

Of the remaining $500 \mathrm{mLs}$ of sample from each tank, $1 \mathrm{~mL}$ of well-mixed sample was preserved by adding $250 \mu \mathrm{L}$ of sugared buffered formalin for future identification and counting under the microscope. Algal density samples were counted within five months of preservation using hemacytometers on standard compound Olympus light microscopes at a magnification of up to $40 \times$. Algal enumeration proceeded by counting 400 cells or four full grids of the hemacytomer (a total volume of $3.6 \mu \mathrm{L}$ ), whichever came first, for each species in each sample. Cell counts were then standardized by the volume counted to achieve cell density estimates $\left(\right.$ cells $\left.\cdot \mathrm{mL}^{-1}\right)$. Cell densities were also multiplied by measured cell biovolumes for each species in order to estimate the total biovolume made up by each species in each community. The cell biovolumes were measured using a FlowCAM (model no. C70).

Up to $50 \mathrm{~mL}$ 's of sampled culture from each tank were then filtered through dry, premassed $47 \mathrm{~mm}$ Whatman GFF filters 
for an estimation of total dry algal biomass. The volume of sample filtered was recorded, filters were dried in a drying oven at $60{ }^{\circ} \mathrm{C}$ for up to 1 week (or until no further mass loss was detected), and the filters were postmassed to estimate total dry biomass. The biomass was divided by the volume of sample filtered to obtain a biomass density in $\mathrm{mg} \cdot \mathrm{L}^{-1}$.

HTL Measurement of Biocrude. We performed all HTL reactions in $1.67 \mathrm{~mL}$ batch reactors built using 3/8-in. 316-stainless-steel Swagelok tube fittings. We loaded all reactors with $48 \mathrm{mg}$ of dry algae and $910 \mathrm{mg}$ of deionized water. These loadings correspond to a 5 wt \% algae loading and enough liquid water to occupy $95 \%$ of the reactor volume at $350{ }^{\circ} \mathrm{C}$, the HTL temperature. The loaded reactors were placed in a sand bath (Techne IFB-51 with a Eurotherm 3216 PID controller) and preheated to $350{ }^{\circ} \mathrm{C}$ for $20 \mathrm{~min}$. After $20 \mathrm{~min}$, we removed reactors from the sand bath, quenched them in a water bath at room temperature for $5 \mathrm{~min}$, and returned them to ambient air for $15 \mathrm{~min}$.

We collected products in a manner similar to procedures employed previously. ${ }^{44}$ In brief, we poured the contents of each vial into a glass conical tube and used $9 \mathrm{~mL}$ of dichloromethane to rinse each reactor and collect remaining products. We vortexed and then centrifuged the conical tube at $3000 \mathrm{rpm}$ for $10 \mathrm{~s}$ and 500 relative centrifugal force (rcf) for $1 \mathrm{~min}$, respectively. The products generally partitioned into an organic, an aqueous, and a solid phase. After transferring the organic phase to another glass vial by pipet, we centrifuged the original glass conical tube again at $1500 \mathrm{rcf}$ for $3 \mathrm{~min}$. We then transferred the aqueous phase from the conical tube to a small plastic vial, leaving only the solid phase behind in the conical tubes. We dried the organic and solid phases using a Labconco RapidVap Vortex evaporator set to a block temperature of $35{ }^{\circ} \mathrm{C}$ with a $\mathrm{N}_{2}$ pressure of 25 psi for 65 and $30 \mathrm{~min}$, respectively. We measured and recorded the mass of each phase, with the biocrude defined as the product remaining after solvent removal from the organic phase. We calculated biocrude yield as the mass of biocrude divided by the initial dry algal biomass. We multiplied these biocrude yield measurements ( $\mathrm{mg}$ biocrude $\cdot \mathrm{mg}$ dry algae ${ }^{-1}$ ) by the biomass density (mg dry algae $\left.\cdot \mathrm{L}^{-1}\right)$ to obtain biocrude production $\left(\mathrm{mg}\right.$ biocrude $\left.\cdot \mathrm{L}^{-1}\right)$. Only reactions with total mass recoveries falling within three standard deviations of the global mean of $83.7 \pm 6.1 \%$ were retained in the reported statistical analyses. Samples with inadequate biomass for the HTL reaction were also necessarily excluded.

Data Analysis. We calculated the mean and coefficient of variation $(\mathrm{CV})$ of biocrude production over the 7-week experiment for each tank:

$$
\mathrm{CV}=\frac{\mathrm{SD}}{\mu}
$$

Where, SD is the standard deviation of biocrude production $\left(\mathrm{mg} \cdot \mathrm{L}^{-1}\right)$ and $\mu$ is the mean biocrude production over the 7-week experiment. For 531 out of 2610 samples, there was insufficient biomass harvested from a tank in order to run an HTL reaction, and for 25 samples, the HTL estimate did not meet quality standards due to human or technical errors. In these cases, we calculated the biocrude production for the missing time point by estimating the mean biocrude yield (mg biocrude $\cdot \mathrm{mg}$ algae ${ }^{-1}$ ) across the remaining time points for that tank, and multiplied the mean yield by the measured biomass production for that time point (mg dry algae $\cdot \mathrm{L}$ of culture ${ }^{-1}$ ). This prevented loss of low biocrude estimates because the biomass data set was complete for the experiment, and biocrude yield was relatively stable within species compositions over time, varying by, on average, less than 2 wt \%.

To determine the probability of dropping below $30 \mathrm{mg} \cdot \mathrm{L}^{-1}$ of biomass as a function of species richness, we created a bootstrap distribution within each level of species richness by sampling the biomass values for each level of species richness with replacement across all species compositions, replicates, and time points. Bootstrapped distributions contained 10000 resampled biomass estimates. Using this distribution, we calculated the probability of the biomass dropping below $30 \mathrm{mg} \cdot \mathrm{L}^{-1}$. We repeated this 1000 times and estimated a mean and standard error of this bootstrapped probability to test whether the probability differed as a function of species richness and temperature treatment.

We used general linear models and linear mixed effects models to test for effects of species richness, species composition (i.e., the particular combination within a given level of richness), temperature treatment, and both the interactions between species richness and species composition and temperature, on the mean and $\mathrm{CV}$ of biocrude production, as well as the probability of biomass dropping below $30 \mathrm{mg} \cdot \mathrm{L}^{-1}$ (hereafter " $P<30$ "). The general linear models were fit using gls() in $\mathrm{R}$ and the linear mixed effects models were fit using the lme() function. In both cases, we used models with variances weighted by species composition, because variances were not equal across treatments. ${ }^{45} \mathrm{We}$ included block as a random effect in the linear mixed effects models for each response variable. We then compared the AIC values of the general linear model against the linear mixed effects model using a log-likelihood ratio test and reported the results of the best model.

We used estimates of the total biovolume of each species in a community to understand how changes in species contributions to community-level biomass affected biomass production and variability. However, due to the time-series nature of the data, and the exorbitant number of samples, we only counted samples for monocultures, 2-species, and 6-species compositions. Overall, total biomass and total biocrude were strongly correlated $(r=0.98, P<0.0001)$. As a result, we assumed that species' contributions to total biomass and hence biocrude could be approximated by looking at their population-level biovolumes. For both the 2- and 6-species communities, we then determined whether each species contributed more or less to community-level biovolume than expected based on an additive expectation-i.e. each species contributes $\left[\frac{1}{\mathrm{SR}} \times\right.$ its monoculture biovolume $]$.

We calculated the CV, mean, and standard deviation of community-level biovolume over time to determine how species richness affected the stability of biovolume, and therefore biomass and biocrude production. Species' population-level biovolumes can contribute to the $\mathrm{CV}$ of community-level biovolume by affecting (1) the mean total biovolume of the community $(\mu)$ and (2) the standard deviation (SD) of the total biovolume of the community (eq 1 above). As the mean community biovolume $(\mu)$ increases, the $\mathrm{CV}$ of the total community biovolume declines. Contrary to $\mu$, when the SD of community-level biovolume increases, the $\mathrm{CV}$ increases. The SD of community biovolume, however, depends on two things: (1) the sum of variances of individual species' population-level biovolumes over time $\left(\sum \sigma_{i}^{2}\right)$ and (2) the synchrony of the biovolume fluctuations among species in the community. ${ }^{28-30,33}$ We estimated synchrony as in the work of Gross et al.: ${ }^{30}$

$$
\text { synchrony }=\frac{1}{N} \cdot \sum_{i} \operatorname{corr}\left(Y_{i}, \sum_{\neq i} Y_{j}\right)
$$


Where $\operatorname{corr}\left(Y_{i}, \sum_{\neq i} Y_{j}\right)$ is the correlation of the biovolume of species $i$ with the summed biovolume of all other species in the community. If population-level biovolumes become individually less stable, then the summed variance $\left(\sum \sigma_{i}^{2}\right)$ will increase, the SD will increase, and the CV will increase, leading to lower stability. Synchrony in population-level biovolume fluctuations (synchrony $>0$ ) will increase the $\mathrm{SD}$, while asynchrony (synchrony $\leq 0$ ) will decrease the $\mathrm{SD}$, decrease the $\mathrm{CV}$, and increase the stability of community-level biovolume.

We removed four tanks from all statistical analyses because more than $5 \%$ of the community biovolume was made up of an algal contaminant (i.e., a species not inoculated into that tank) on the final week of the experiment, week 7 .

\section{RESULTS}

While species richness had a significant effect on biocrude production (Table 1), increasing richness resulted in reduced

Table 1. ANOVA Table Showing Effects of Species Richness (SR), Temperature Treatment (Temperature), Species Composition (CompositionlSR), and the Interactions between Diversity and Temperature Treatment (SR·Temperature), and Species Composition and Temperature Treatment (CompositionlSR $\cdot$ Temperature) on the Mean Biocrude Production $\left(\mathrm{mg}^{\cdot} \mathrm{L}^{-1}\right)$ over Time ${ }^{a}$

\begin{tabular}{llrc}
\multicolumn{1}{c}{ effect } & \multicolumn{2}{c}{ F-value } & $P$-value \\
SR & $F_{1,292}$ & 16.49 & 0.0001 \\
temperature & $F_{1,292}$ & 5.38 & 0.02 \\
composition|SR & $F_{14,292}$ & 4.02 & $<0.0001$ \\
SR.temperature & $F_{1,292}$ & 0.69 & 0.41 \\
compositionlSR.temperature & $F_{14,292}$ & 0.87 & 0.60
\end{tabular}

${ }^{a}$ This model was a linear mixed effects model, containing block as a random effect.

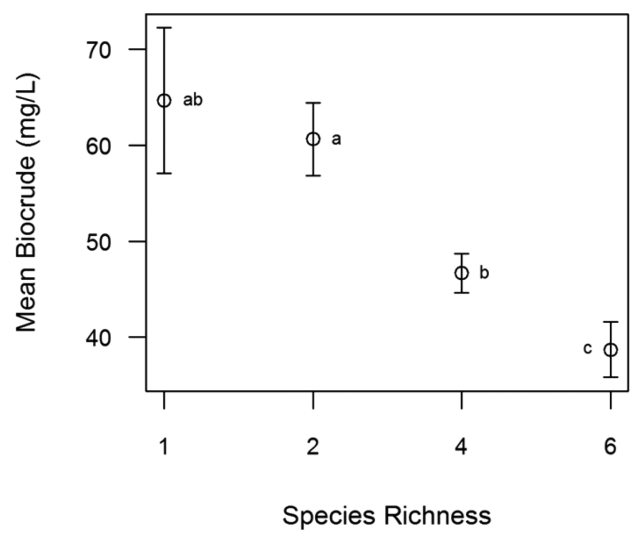

Figure 1. Effect of species richness on mean biocrude production over time. Points indicate the mean biocrude production per week $\left(\mathrm{mg} \cdot \mathrm{L}^{-1}\right.$ ) across all compositions of the same species diversity, averaged over 7 weeks. Letters indicate significant differences among diversity treatments according to Tukey's posthoc tests $(\alpha=0.05)$. Error bars indicate standard error.

production, which was contrary to our prediction $\left(\overline{\bar{Y}}_{\mathrm{P}}>\overline{\bar{Y}}_{\mathrm{M}}\right.$, Figure 1). The average biocrude production declined by $40 \%$ as species richness increased from 1 to 6 species in mixture (Figure 1). Our analysis of population and community biovolumes indicated that most species achieved lower biovolumes when they were in polycultures relative to monocultures, and this was most pronounced in the most diverse communities (SI Figure 4).
Temperature variability significantly affected biocrude production (Table 1), with the constant temperature treatment producing a mean of $51.85 \mathrm{mg} \cdot \mathrm{L}^{-1}$ per week $( \pm 2.82$ s.e. $)$ and the variable temperature treatment producing $55.75 \mathrm{mg} \cdot \mathrm{L}^{-1}$ of biocrude per week $( \pm 3.08$ s.e.). Temperature variability did not affect the size of the species richness effect on biocrude production (Table 1). Within each level of species richness, there were particular species or species combinations that had significantly different levels of production (Table 1). The monoculture with the greatest biocrude production was Selenastrum capricornutum, with a mean of $140.66 \mathrm{mg} \cdot \mathrm{L}^{-1} \pm$ 11.72 (s.e.) (composition F, Figure 2). While this species had the highest production on average, it did not produce significantly more biocrude than the second best composition, which was a biculture of Selenastrum capricornutum and Scenedesmus acuminatus (composition DF, Figure 2, Wilcoxon sign rank test $W=69.50, P=0.11$ ). Therefore, while we reject the original prediction that the most productive polyculture produces more biocrude than the most productive monoculture $\left(\bar{Y}_{\text {best } \mathrm{P}}>\bar{Y}_{\text {best } M}\right)$, our data do not support the alternative prediction that monocultures produce any more biocrude than the most productive polyculture $\left(\bar{Y}_{\text {best_M }}>\bar{Y}_{\text {best_p }}\right)$.

Species richness had a significant stabilizing effect on biocrude production over time (SR, Table 2). Polycultures with two and four species were significantly more stable than monocultures (SI Figure 5). However, there was no main effect of the temperature treatment on the $\mathrm{CV}$ of biocrude production (Table 2). While there were no significant interactive effects of the temperature treatment and species richness, or the temperature treatment and species composition on the $\mathrm{CV}$ of biocrude production (Table 2), the $p$-value for the SR-temperature interaction was 0.11 , which is suggestive of a possible effect of temperature. We explored this potential interaction further by analyzing the effects of diversity and composition on the $\mathrm{CV}$ of biocrude production separately in the constant and variable temperature treatments. We found that species richness did tend to stabilize biocrude production over time (i.e., reduced the $\mathrm{CV}$ ) in the variable temperature environment, but not in the constant temperature environment (Figure 3). Specifically, in the variable temperature environment, 2-, 4-, and 6-species polycultures were more stable than monocultures on average (Tukey's posthoc comparisons with monoculture, $P<0.05$ ), but this was not the case in the constant temperature environment (Tukey's posthoc comparisons with monoculture, $P>0.05$, Figure 3 ). In the variable temperature environment, the $\mathrm{CV}$ of biocrude in the average polyculture declined by $29 \%$ relative to monoculture, but there was no significant difference in the constant environment, supporting our hypothesis that diversity would have a greater stabilizing effect in variable than in constant temperature environments $\left(\left[\overline{\mathrm{CV}}_{\mathrm{M}}-\overline{\mathrm{CV}}_{\mathrm{P}}\right]_{\text {variable }}>\left[\overline{\mathrm{CV}}_{\mathrm{M}}-\overline{\mathrm{CV}}_{\mathrm{P}}\right]_{\text {constant }}\right)$.

We used cell counts and biovolume data to determine how the mean, variance, and synchrony of individual species' population-level biovolumes contributed to total communitylevel biovolume and, by extension, biocrude production over time. Trends in the CV of community-level biovolume over time mirrored the trends in $\mathrm{CV}$ of biocrude in response to species richness (SI Figure 6A and B). There was a marginally nonsignificant interaction between the temperature treatment and species richness on the $\mathrm{CV}$ of community-level biovolume (SR.temperature, $F_{1,180}=3.66, P=0.06$ ). This interaction reflects that there was a decline in the $\mathrm{CV}$ of biovolume with species richness in the variable temperature environment, but 


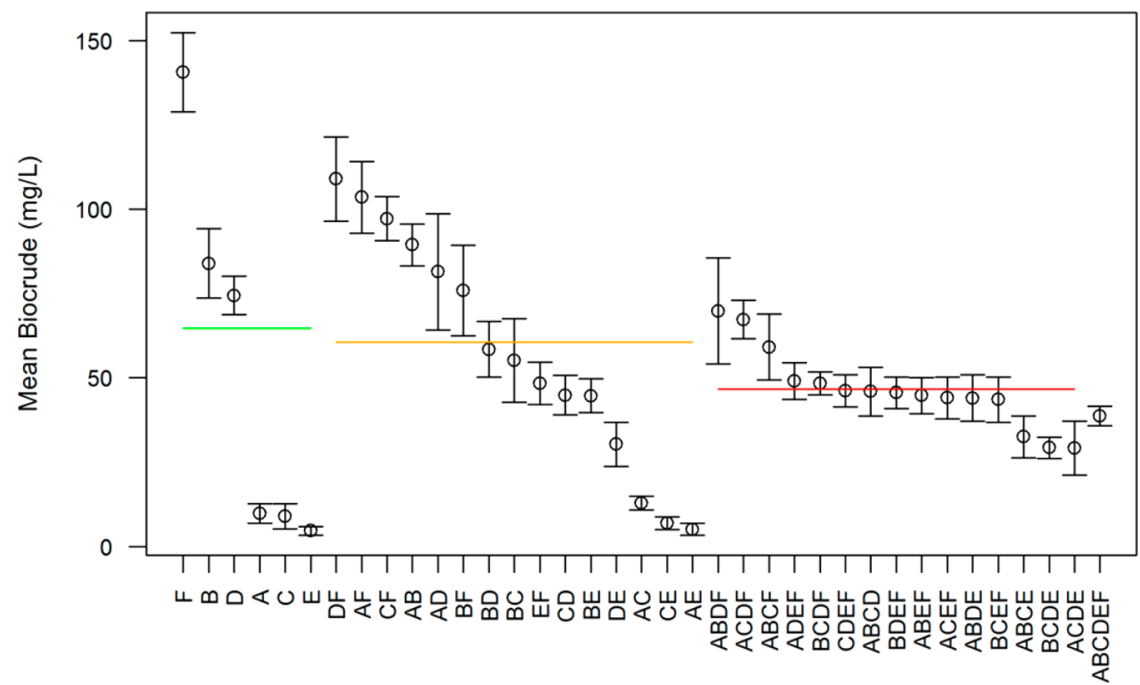

Figure 2. Effect of species composition on mean biocrude production over time. Points indicate the mean biocrude production per week $\left(\mathrm{mg} \cdot \mathrm{L}^{-1}\right)$ across all replicates of the same composition, averaged over 7 weeks. Error bars indicate standard error. Colored lines indicate the mean biocrude for each level of species richness with green indicating monocultures, orange indicating bicultures, and red indicating 4-species cultures.

Table 2. ANOVA Table Showing Effects of Species Richness (SR), Temperature Treatment (Temperature), Species Composition (CompositionlSR), and the Interactions between Diversity and Temperature Treatment (SR. Temperature) and Species Composition and Temperature Treatment (Composition|SR $\cdot$ Temperature) on the $\mathrm{CV}$ of Biocrude over Time ${ }^{a}$

\begin{tabular}{llcc}
\multicolumn{1}{c}{ effect } & \multicolumn{2}{c}{$F$-value } & $P$-value \\
SR & $F_{1,289}$ & 26.58 & $<0.0001$ \\
temperature & $F_{1,289}$ & 2.01 & 0.16 \\
compositionISR & $F_{14,289}$ & 1.18 & 0.29 \\
SR.temperature & $F_{1,289}$ & 2.56 & 0.11 \\
compositionISR-temperature & $F_{14,289}$ & 0.85 & 0.61
\end{tabular}

${ }^{a}$ This was a linear mixed effects model, containing block as a random effect.

not in the constant environment (SI Figure 6A and B). The effect of species richness on the mean and standard deviation of biovolume did not significantly depend on temperature $\left(F_{1,180}=0.18, P=0.67\right.$, and $F_{1,180}=0.51, P=0.48$, respectively). Species richness had a negative main effect on both the mean $\left(F_{1,180}=146.31, P<0.0001\right)$ and standard deviation of biovolume $\left(F_{1,180}=19.49, P<0.0001\right)$. A reduction in the mean biovolume reduces stability, and as a result the stabilizing effect of diversity observed in the variable temperature treatment must have been due to a reduction in the standard deviation of biovolume (SI Figure 6C-E). The reduced standard deviation of biovolume with species richness in the variable environment (though nonsignificant) can be explained by a decline in the summed population level variances with increasing species richness (log-transformed summed variance, $F_{1,180}=13.40$, $P=0.0003$, SI Figure 7B), and not by a decrease in synchrony (SI Figure 7C and D). Thus, the increased biocrude stability with species richness in the variable temperature environment is likely explained by reduced temporal fluctuations of individual species' populations, rather than any form of asynchronous dynamics among different species' populations.

Overall, species composition did not have a significant impact on the CV of biocrude production (Table 2). The monoculture of Selenastrum capricornutum (species F) was the most

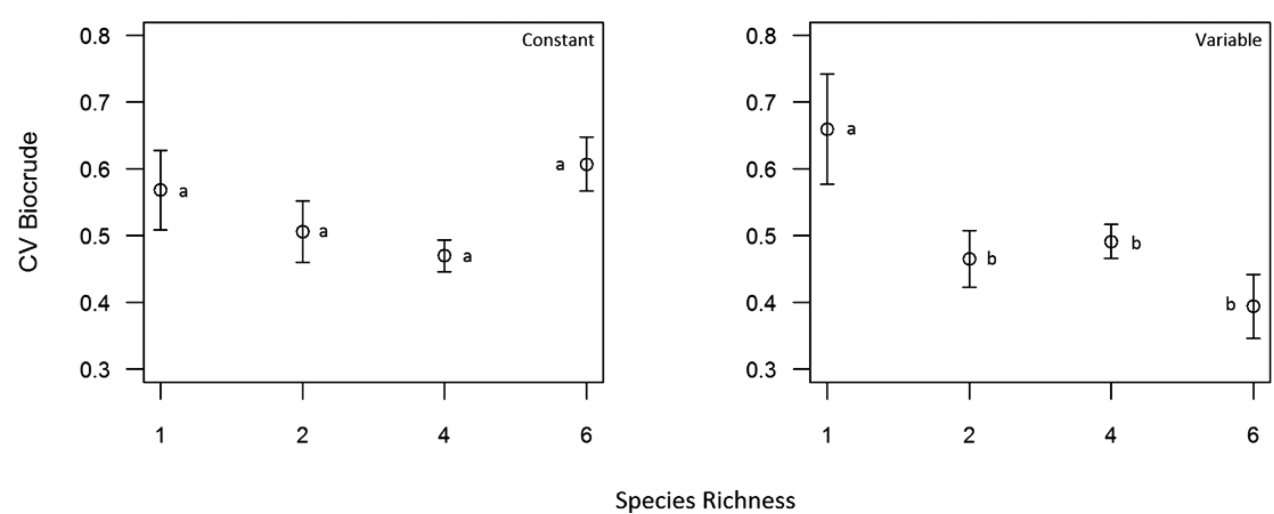

Figure 3. Effect of species richness on the coefficient of variation $(\mathrm{CV})$ of biocrude production over time as a function of temperature treatment. There was no significant effect of species richness in the constant environment $\left(F_{3,156}=1.64, P=0.18\right)$, but there was a significant effect of species richness in the variable environment $\left(F_{3,156}=4.33, P=0.006\right)$. Data points indicate the $\mathrm{CV}$ of biocrude production over 7 weeks $\left(\mathrm{mg} \cdot \mathrm{L}^{-1}\right)$ averaged across all compositions of the same species diversity and temperature treatment. Letters indicate significant differences among diversity treatments according to Tukey's posthoc tests $(\alpha=0.05)$. Error bars represent the standard error. 
stable species composition (SI Figure 8), with an average CV of $0.28 \pm 0.04$ (s.e.), but Chlorella sorokiniana (species B) and Scenedesmus acuminatus (species D) did not have significantly higher CVs (Dunnett's, $P_{\mathrm{B}}=0.99, P_{\mathrm{D}}=0.24$ ). There was no polyculture that had significantly more stable biocrude than $S$. capricornutum (all one-way Dunnett's posthoc comparisons, $P>0.05$, SI Figure 8), rejecting the hypothesis that the most stable polyculture would be more stable than the most stable monoculture $\left(\overline{\mathrm{CV}}_{\text {best_P }}<\overline{\mathrm{CV}}_{\text {best_M }}\right)$. However, many polycultures were not significantly less stable than S. capricornutum, including the biculture of $S$. acuminatus and S. capricornutum (composition DF, SI Figure 8, Wilcoxon sign rank test $W=26.00, P=0.10)$.

Lastly, we tested the prediction that polycultures would have a higher probability of staying above a production failure threshold $\left(30 \mathrm{mg} \cdot \mathrm{L}^{-1}\right)$ than monocultures: $\overline{\operatorname{Pr}\left[Y_{\mathrm{P}}>T^{*}\right]}>$ $\overline{\operatorname{Pr}\left[Y_{M}>T^{*}\right]}$. We found that the bootstrapped probabilities of dropping below the threshold declined with increasing species richness $\left(\mathrm{SR}, F_{3,7992}=14432.30, P<0.0001\right)$, and that this effect was stronger in a variable temperature environment than in a constant temperature environment (SR.temperature $F_{3,7992}=362.75, P<0.0001$, SI Figure 9). As a result, we failed to reject the hypothesis that polycultures are more likely to stay above a production threshold than monocultures $\left(\overline{\operatorname{Pr}\left[Y_{\mathrm{P}}>T^{*}\right]}>\right.$ $\left.\overline{\operatorname{Pr}\left[Y_{M}>T^{*}\right]}\right)$, and adding species richness has a greater stabilizing effect in variable than in constant environments $\left(F_{\text {variable } 3,3996}=8102.50\right.$ versus $\left.F_{\text {constant3,3996 }}=5965.50\right)$. Overall, the six species community in the variable temperature environment had the lowest probability of dropping below $30 \mathrm{mg} \cdot \mathrm{L}^{-1}$ of biomass, at probability of $4 \% \pm 4 \%$ (95\% C.I.) (SI Figure 9).

\section{DISCUSSION}

Growing mixed consortia of species tended to decrease biocrude production relative to the average monoculture, likely as a result of competition among species. However, polycultures were, on average, more stable in their biocrude production when water temperatures were fluctuating through time because diversity reduced the summed variance of species' biovolumes. In addition, polycultures were more reliable than the average monoculture in the sense that they had lower probabilities of dropping below a threshold that might lead to crop failure. Although the typical polyculture was more stable and reliable than the typical monoculture, there were only a small subset of polycultures able to equal (but none able to exceed) the biocrude production and stability of the single "best" monoculture (S. capricornutum).

To date, few studies have investigated the role that species diversity plays in increasing or stabilizing biofuel production by algae. Our results are most consistent with that of Bhattacharjee and Siemann, ${ }^{37}$ who showed that select monocultures are able to produce abundant and stable supplies of biofuel. Given the particular environmental conditions used in this experiment, a monoculture of $S$. capricornutum was the most productive and stable. However, there were bicultures, some containing S. capricornutum that were equally productive and stable. Moreover, while all of the monocultures have been previously identified as candidates for biofuel production, some species were highly unproductive and unstable (namely Ankistrodesmus falcatus, Scenedesmus ecornis, and Pediastrum duplex). Since the average biculture was not significantly less productive than the average monoculture but was significantly more stable, when the best monoculture for a given set of environmental conditions is unknown, it may be better to select a biculture of biofuels candidate species than to select a monoculture. ${ }^{10}$ Due to their higher average stability over time, the use of bicultures may help to limit the costs of production that arise due to unwanted pond crashes and culture restarts.

Our results contrast with those of Shurin et al. ${ }^{10,11}$ and Stockenreiter et al. ${ }^{35,36}$ who found positive effects of species or functional diversity on lipid production or biovolume. Stockenreiter et al. $^{36}$ found that lipid production was positively affected by species richness in biodiversity experiments using lab-assembled, as well as natural communities, of freshwater algae. Shurin et al. ${ }^{10,11}$ found that some multispecies compositions were capable of producing even more biovolume than the most productive monocultures, but our results indicate that no polyculture was able to outperform the best-performing monoculture. We also acknowledge that our results contradict the consensus of research on the impact of biodiversity on ecosystem functioning, which has shown that diversity generally has a positive and saturating impact on ecosystem functions such as biomass production and resource use efficiency. ${ }^{16-18,46}$ These contradictions could be due to the limited species pool selected for our experiments. We included only freshwater green algae that have been previously considered for biofuels production in past research. ${ }^{7,39-41}$ By comparison, prior studies selected species from many taxonomic and functional groups (including cyanobacteria and diatoms), which could be more in line with typical biodiversity experiments. ${ }^{11,36}$ As a result, the species that we selected may represent a limited portion of ecological trait space, and by including a greater set of taxonomic groups it is possible that prior studies captured the trait variation needed for niche partitioning and overyielding. This interpretation is supported by a recent study on an open raceway pond showing that periods of relatively high naturally occurring microbial (bacterial and eukaryotic) genetic diversity, tended to be associated with high biomass production and stability. ${ }^{47}$ As a result, we would recommend further experiments to test whether biodiversity sampled from a broader selection of taxonomic and functional groups, including locally adapted taxa, might be scaled-up to provide the yield benefits which are often observed in smaller-scale biodiversity experiments.

We used individual species' biovolume data to investigate the mechanisms driving the influence of biodiversity on mean biocrude production and stability. We found that average biocrude production tended to decline with biodiversity, and this was likely because the four most productive species in monoculture tended to underyield in polyculture, with the degree of underyielding becoming more extreme in 6-species communities than in bicultures (SI Figure 4). This result is in line with prior studies demonstrating that underyielding may not be uncommon in diverse algal communities. ${ }^{11,48,49}$ The number of studies investigating the mechanisms by which diversity influences biomass stability over time is still limited, but a recent meta-analysis of algal microcosm studies showed that diversity tends to have approximately equal positive influences on both the mean and variance of community-level biomass and therefore no net effect on biomass stability via these mechanisms. ${ }^{30}$ However, they also found that synchrony declines with diversity, which has a stabilizing effect. By contrast, we found that both the mean and summed variance of community biovolume declined with diversity, and that population-level synchrony was not affected by diversity (SI Figures 6 and 7). 
In our study, a reduced summed variance with increasing diversity was the only mechanism which could cause the reduced biocrude variability in the variable temperature treatment. We recommend that similar studies are repeated with other species pools and sources of environmental variation to assess the generality of our findings and to resolve the contrast with prior results.

In conclusion, we have shown that species diversity has the potential to stabilize biocrude production over time, particularly in the presence of environmental temperature variability. In contrast to prior studies, however, we did not find that diversity tends to increase biocrude production on average, or beyond the best monoculture. When productive and stable monocultures can be identified, they may be the best option for scaling-up biocrude production. However, when the best monoculture is unknown, many mixtures, and in particular bicultures, may provide moderate levels of production that are much more stable over time than the average monoculture. This may help to mitigate the high costs incurred by culture crashes and restarts. In this study, we investigated the effect of diversity on biocrude production and stability in the face of a single type of environmental variability-temperature-in a fairly controlled environment with the aim of mimicking only one aspect of natural environmental variation. However, diversity may have greater importance in maximizing and stabilizing biocrude production in truly open systems where species face a number of types of environmental and biotic variability. ${ }^{10,12,50,51}$ Future research should focus on identifying the most serious agents of crop losses in open systems, and identifying the suites of species that can resist these various threats. In years where crops are lost, it may prove useful to identify and study species that remain or are able to invade after the focal crop species is lost. Such natural experiments may be instructive for future attempts to engineer productive and stable communities for fuel production.

\section{ASSOCIATED CONTENT}

\section{(S) Supporting Information}

The Supporting Information is available free of charge on the ACS Publications website at DOI: 10.1021/acs.est.6b03256.

Nine figures and three tables (PDF)

\section{AUTHOR INFORMATION}

\section{Corresponding Author}

*Phone: +41 58765 5667. Fax: +41 58765 5802. E-mail: anita.narwani@eawag.ch.

\section{ORCID}

Phillip E. Savage: 0000-0002-7902-3744

\section{Notes}

The authors declare no competing financial interest.

\section{ACKNOWLEDGMENTS}

We would like to thank a number of people for their help in running the experiment in order of contribution (i.e. greatest first): L. Wolterink, B. Gregory, M. Busch, J. Fenno, A. Sedlar, E. Moser, and A. Davila. We would also like to thank a number of people for their assistance in processing HTL samples, in order of contribution: A. Hoang, C. Koss, K. Lindstrom, M. C. Meader, D. Mees, N. Arunrattanamook, H. Chen, G. Teri, L. Luo, C. S. Chan, W. Tandar, L. Miller, and M. Irish. This work was funded by a grant from the Emerging Frontiers in Research Innovation from the U.S. National Science Foundation (award no. 1332342) to B.J.C. and P.S.

\section{REFERENCES}

(1) National Algal Biofuels Technology Roadmap; U.S. Department of Energy, Office of Energy Efficiency and Renewable Energy, Biomass Program, 2010; https://www1.eere.energy.gov/bioenergy/pdfs/algal_ biofuels_roadmap.pdf.

(2) Chisti, Y. Biodiesel from microalgae beats bioethanol. Trends Biotechnol. 2008, 26, 126-131.

(3) Chisti, Y. Biodiesel from microalgae. Biotechnol. Adv. 2007, 25, 294-306.

(4) Georgianna, D. R.; Mayfield, S. P. Exploiting diversity and synthetic biology for the production of algal biofuels. Nature 2012, 488, 329-335.

(5) Sustainable Development of Algal Biofuels in the United States; Committee on the Sustainable Development of Algal Biofuels, Board of Agriculture and Natural Resources, Division on Earth and Life Studies, Board on Energy and Environmental Systems, Division on Engineering and Physical Sciences, National Research Council, National Academy Press: Washington, D.C., USA, 2012.

(6) Pienkos, P. T.; Darzins, A. The promise and challenges of microalgal-derived biofuels. Biofuels, Bioprod. Biorefin. 2009, 3, 431440.

(7) NREL Close-Out Report. NREL/TP-580-24190. A Look Back at the U.S. Department of Energy's Aquatic Species Program-Biodiesel from Algae; Sheehan, J., Dunahay, T., Benemann, J., Roessler, P., Eds.; U.S. Department of Energy, National Renewable Energy Laboratory: Golden, CO, 1998; http://www.nrel.gov/docs/legosti/fy98/24190. pdf.

(8) Benemann, J. Microalgae for Biofuels and Animal Feeds. Energies 2013, 6, 5869-5886.

(9) Chisti, Y.; Yan, J. Energy from algae: Current status and future trends Algal biofuels - A status report. Appl. Energy 2011, 88, 32773279.

(10) Shurin, J. B.; Abbott, R. L.; Deal, M. S.; Kwan, G. T.; Litchman, E.; McBride, R. C.; Mandal, S.; Smith, V. H. Industrial-strength ecology: trade-offs and opportunities in algal biofuel production. Ecol. Lett. 2013, 16, 1393-1404.

(11) Shurin, J. B.; Mandal, S.; Abbott, R. L. Trait diversity enhances yield in algal biofuel assemblages. J. Appl. Ecol. 2014, 51, 603-611.

(12) Smith, V. H.; Crews, T. Applying ecological principles of crop cultivation in large-scale algal biomass production. Algal Res. 2014, 4, 23-34.

(13) Bull, J. J.; Collins, S. Algae for biofuel: Will the evolution of weeds limit the enterprise? Evolution 2012, 66, 2983-2987.

(14) Day, J. G.; Slocombe, S. P.; Stanley, M. S. Overcoming biological constraints to enable the exploitation of microalgae for biofuels. Bioresour. Technol. 2012, 109, 245-251.

(15) Lindemann, S. R.; Bernstein, H. C.; Song, H.-S.; Fredrickson, J. K.; Fields, M. W.; Shou, W.; Johnson, D. R.; Beliaev, A. S. Engineering microbial consortia for controllable outputs. ISME J. 2016, 10, 20772084.

(16) Schmid, B.; Balvanera, P.; Cardinale, B. J.; Godbold, J.; Pfisterer, A. B.; Raffaelli, D.; Solan, M.; Srivastava, D. Consequences of species loss for ecosystem functioning: a meta-analysis of data from biodiversity experiments. In Biodiversity, Ecosystem Functioning, \& Human Wellbeing; Naeem, S., Bunker, D. E., Hector, A., Loreau, M., Perrings, C., Eds.; Oxford University Press: New York, 2009; pp 1429.

(17) Cardinale, B. J.; Matulich, K. L.; Hooper, D. U.; Byrnes, J. E.; Duffy, E.; Gamfeldt, L.; Balvanera, P.; O’Connor, M. I.; Gonzalez, A. The functional role of producer diversity in ecosystems. Am. J. Bot. 2011, 98, 572-592.

(18) Cardinale, B. J.; Duffy, J. E.; Gonzalez, A.; Hooper, D. U.; Perrings, C.; Venail, P.; Narwani, A.; Mace, G. M.; Tilman, D.; Wardle, D. A.; Kinzig, A. P.; Daily, G. C.; Loreau, M.; Grace, J. B.; Larigauderie, A.; Srivastava, D. S.; Naeem, S. Biodiversity loss and its impact on humanity. Nature 2012, 486, 59-67.

(19) Hooper, D. U.; Adair, E. C.; Cardinale, B. J.; Byrnes, J. E. K.; Hungate, B. A.; Matulich, K. L.; Gonzalez, A.; Duffy, J. E.; Gamfeldt, 
L.; O'Connor, M. I. A global synthesis reveals biodiversity loss as a major driver of ecosystem change. Nature 2012, 486, 105-U129.

(20) Hooper, D. U.; Chapin, F. S.; Ewel, J. J.; Hector, A.; Inchausti, P.; Lavorel, S.; Lawton, J. H.; Lodge, D. M.; Loreau, M.; Naeem, S.; Schmid, B.; Setala, H.; Symstad, A. J.; Vandermeer, J.; Wardle, D. A. Effects of biodiversity on ecosystem functioning: A consensus of current knowledge. Ecol. Monogr. 2005, 75, 3-35.

(21) Haddad, N. M.; Crutsinger, G. M.; Gross, K.; Haarstad, J.; Tilman, D. Plant diversity and the stability of foodwebs. Ecol. Lett. 2011, 14, 42-46.

(22) Huston, M. A. Hidden treatments in ecological experiments: Reevaluating the ecosystem function of biodiversity. Oecologia 1997, 110, 449-460.

(23) Tilman, D. The ecological consequences of changes in biodiversity: A search for general principles. Ecology 1999, 80, 1455-1474.

(24) Ives, A. R.; Gross, K.; Klug, J. L. Stability and variability in competitive communities. Science 1999, 286, 542-544.

(25) Doak, D. F.; Bigger, D.; Harding, E. K.; Marvier, M. A.; O'Malley, R. E.; Thomson, D. The statistical inevitability of stabilitydiversity relationships in community ecology. Am. Nat. 1998, 151, 264-276.

(26) Tilman, D. Biodiversity: Population versus ecosystem stability. Ecology 1996, 77, 350-363.

(27) Tilman, D.; Lehman, C. L.; Bristow, C. E. Diversity-stability relationships: Statistical inevitability or ecological consequence? Am. Nat. 1998, 151, 277-282.

(28) Cottingham, K. L.; Brown, B. L.; Lennon, J. T. Biodiversity may regulate the temporal variability of ecological systems. Ecol. Lett. 2001, $4,72-85$.

(29) Loreau, M.; de Mazancourt, C. Biodiversity and ecosystem stability: a synthesis of underlying mechanisms. Ecol. Lett. 2013, 16, $106-115$.

(30) Gross, K.; Cardinale, B. J.; Fox, J. W.; Gonzalez, A.; Loreau, M.; Polley, H. W.; Reich, P. B.; van Ruijven, J. Species richness and the temporal stability of biomass production: A new analysis of recent biodiversity experiments. Am. Nat. 2014, 183, 1-12.

(31) Yachi, S.; Loreau, M. Biodiversity and ecosystem productivity in a fluctuating environment: The insurance hypothesis. Proc. Natl. Acad. Sci. U. S. A. 1999, 96, 1463-1468.

(32) Ives, A. R.; Klug, J. L.; Gross, K. Stability and species richness in complex communities. Ecol. Lett. 2000, 3, 399-411.

(33) Lehman, C. L.; Tilman, D. Biodiversity, stability, and productivity in competitive communities. Am. Nat. 2000, 156, 534552.

(34) Narwani, A.; Mazumder, A. Bottom-up effects of species diversity on the functioning and stability of food webs. J. Anim. Ecol. 2012, 81, 701-713.

(35) Stockenreiter, M.; Graber, A. K.; Haupt, F.; Stibor, H. The effect of species diversity on lipid production by micro-algal communities. $J$. Appl. Phycol. 2012, 24, 45-54.

(36) Stockenreiter, M.; Haupt, F.; Graber, A.-K.; Seppala, J.; Spilling, K.; Tamminen, T.; Stibor, H. Functional group richness: Implications of biodiversity for light use and lipid yield in microalgae. J. Phycol. 2013, 49, 838-847.

(37) Bhattacharjee, M.; Siemann, E. Low algal diversity systems are a promising method for biodiesel production in wastewater fed open reactors. Algae 2015, 30, 67-79.

(38) Smith, V. H.; Sturm, B. S.; Denoyelles, F. J.; Billings, S. A. The ecology of algal biodiesel production. Trends Ecol. Evol. 2010, 25, 301309.

(39) Rodolfi, L.; Zittelli, G. C.; Bassi, N.; Padovani, G.; Biondi, N.; Bonini, G.; Tredici, M. R. Microalgae for oil: strain selection, induction of lipid synthesis and outdoor mass cultivation in a low-cost photobioreactor. Biotechnol. Bioeng. 2009, 102, 100-112.

(40) Mata, T. M.; Martins, A. A.; Caetano, N. S. Microalgae for biodiesel production and other applications: A review. Renewable Sustainable Energy Rev. 2010, 14, 217-232.
(41) Griffiths, M. J.; Harrison, S. T. L. Lipid productivity as a key characteristic for choosing algal species for biodiesel production. J. Appl. Phycol. 2009, 21, 493-507.

(42) Chisti, Y. Raceways-based production of algal crude oil. Green 2013, 3, 195-216.

(43) Andersen, R., Ed. Algal culturing techniques; Academic Press: New York, 2005.

(44) Valdez, P. J.; Nelson, M. C.; Wang, H. Y.; Lin, X. N.; Savage, P. E. Hydrothermal liquefaction of Nannochloropsis sp.: Systematic study of process variables and analysis of the product fractions. Biomass Bioenergy 2012, 46, 317-331.

(45) Zuur, A. F., Ieno, E. N., Walker, N. J., Saveliev, A. A., Smith, G. M. Mixed effects models and extensions in Ecology with R; Springer Publishing: New York, 2009.

(46) Balvanera, P.; Pfisterer, A. B.; Buchmann, N.; He, J.-S.; Nakashizuka, T.; Raffaelli, D.; Schmid, B. Quantifying the evidence for biodiversity effects on ecosystem functioning and services. Ecol. Lett. 2006, 9, 1146-1156.

(47) Beyter, D.; Tang, P.-Z.; Becker, S.; Hoang, T.; Bilgin, D.; Lim, Y. W.; Peterson, T. C.; Mayfield, S.; Haerizadeh, F.; Shurin, J. B.; Bafna, V.; McBride, R. Diversity, productivity, and stability of an industrial microbial ecosystem. Appl. Environ. Microbiol. 2016, 82, 2494-2505.

(48) Weis, J. J.; Madrigal, D. S.; Cardinale, B. J. Effects of algal diversity on the production of biomass in homogeneous and heterogeneous nutrient environments: a microcosm experiment. PLoS One 2008, 3, e2825.

(49) Schmidtke, A.; Gaedke, U.; Weithoff, G. A mechanistic basis for underyielding in phytoplankton communities. Ecology 2010, 91, 212221.

(50) Hector, A.; Bagchi, R. Biodiversity and ecosystem multifunctionality. Nature 2007, 448, 188-190.

(51) Gamfeldt, L.; Snall, T.; Bagchi, R.; Jonsson, M.; Gustafsson, L.; Kjellander, P.; Ruiz-Jaen, M. C.; Froberg, M.; Stendahl, J.; Philipson, C. D.; Mikusinski, G.; Andersson, E.; Westerlund, B.; Andren, H.; Moberg, F.; Moen, J.; Bengtsson, J. Higher levels of multiple ecosystem services are found in forests with more tree species. Nat. Commun. 2013, 4, 1340. 